\title{
Hospital Costs of Adverse Events in Patients with Metastatic Colorectal Cancer*
}

\author{
Alex Z. Fu' ${ }^{1}$ Zhongyun Zhao ${ }^{2 \#}$, Song Wang ${ }^{2}$, Beth Barber ${ }^{2}$, Gordon G. Liu ${ }^{3}$ \\ ${ }^{1}$ Cancer Prevention and Control Program, Lombardi Comprehensive Cancer Center, Georgetown University, Washington DC, USA; \\ ${ }^{2}$ Amgen Inc., One Amgen Center Drive, Thousand Oaks, USA; ${ }^{3}$ Guanghua School of Management, Peking University, Beijing, \\ China. \\ Email: "Zhongyun@amgen.com
}

Received September $26^{\text {th }}, 2012$; revised October $28^{\text {th }}, 2012$; accepted November $5^{\text {th }}, 2012$

\begin{abstract}
Background: Monoclonal antibody treatments for metastatic colorectal cancer (mCRC) have distinct treatment-related safety profiles. This study aimed to elucidate the hospitalisation costs of adverse events (AEs) commonly associated with monoclonal antibodies when administered to patients with mCRC. Methods: This study extracted data for patients newly diagnosed with mCRC from a large US claims database from January 2005 to June 2008. The first distant metastasis diagnosis date was defined as the index date. Main outcomes were length of hospital stay (days) and hospitalisation costs (2010 US\$) for AEs (identified by primary discharge diagnoses). All analyses are presented descriptively. Results: The study population (aged $\geq 18$ years; $\mathrm{n}=12,648$ ) was balanced according to gender and was mainly aged 50 years or older $(90.1 \%)$. Most patients had colon cancer $(70.1 \%)$ as opposed to rectal cancer. Gastrointestinal (GI) perforation incurred the longest median length of stay (11.5 days) for hospitalisations, followed by wound-healing complications ( 7 days), arterial and venous thromboembolism (5.5 and 4 days, respectively), and congestive heart failure (4 days). The highest inpatient cost per event was for GI perforations (mean $\$ 66,224$ and median $\$ 34,027$ ), followed by arterial thromboembolism $(\$ 40,992$ and $\$ 18,587)$, wound-healing complications $(\$ 36,440$ and $\$ 21,163)$, interstitial lung disease $(\$ 26,705$ and $\$ 19,111)$ and acute myocardial infarction $(\$ 22,395$ and $\$ 15,223)$. Skin toxicity (mean $\$ 6475$ and median \$6110) and hypertension (\$14,108 and \$6047) were associated with relatively low costs. Conclusions: Hospital costs for monoclonal antibody treatment-related AEs in patients with mCRC vary greatly. This study provides source data for economic evaluations of head-to-head comparisons of monoclonal antibody treatments.
\end{abstract}

Keywords: Monoclonal Antibody; Metastatic Colorectal Cancer; Adverse Events; mCRC; Hospital Costs

\section{Introduction}

Colorectal cancer (CRC) is the third most common cancer diagnosed in both men and women in the USA [1]. As such, CRC has been associated with the second highest cancer costs to Medicare (the US Social Security Administration reimbursement programme), with net costs increasing at later stages of diagnosis [2]. Studies have shown that substantial differences in costs of care for patients with metastatic CRC (mCRC) were found between $\mathrm{mCRC}$ disease phases. Inpatient care was the key driver of medical care in the diagnostic and death phases compared with outpatient care in the treatment phase [3].

Improvements in the survival of patients with $\mathrm{mCRC}$ have been made over the past two decades with recent advances in systemic therapies, including monoclonal

*Alex Z. Fu and Gordon G. Liu received funding from Amgen Inc. Zhongyun Zhao, Song Wang and Beth Barber are all employees of Amgen Inc.

${ }^{\#}$ Corresponding author. antibody treatments targeting the vascular endothelial growth factor (VEGF) such as bevacizumab or epidermal growth factor receptor (EGFR) such as cetuximab or panitumumab $[4,5]$. Although monoclonal antibodies have improved outcomes, they have treatment-related toxicities. The safety profile of an anti-EGFR antibody however, is distinct from that of an anti-VEGF antibody. According to the "Warnings and precautions" section of the US product information (PI) documents [6-8] and the "Special warnings and precautions for use" section of the European Medicines Agency (EMA) Summary of Product Characteristics (SmPC) [9-11], severe adverse events (SAEs) associated with the anti-EGFR antibodies, cetuximab and panitumumab, include serious infusion reactions, pulmonary toxicity, dermatologic toxicity, hypomagnesaemia and other electrolyte abnormalities. In contrast, the anti-VEGF antibody, bevacizumab, is associated with the following SAEs: gastrointestinal (GI) perforations, surgery and wound-healing complications, 
haemorrhage, non-GI fistula formation, arterial thromboembolism and hypertension [6].

Adverse events (AEs) associated with mCRC treatments have the potential to impact healthcare resource utilisation and the overall cost of patient care [12]. However, few studies have evaluated the direct costs associated with treatment-related AEs of monoclonal antibodies in patients with mCRC. This claims-based cohort study aimed to elucidate the hospitalisation costs of AEs, documented in the literature and regulatory agencies approved labels as being associated with bevacizumab, cetuximab or panitumumab when administered to patients with mCRC, in order to provide source data for future cost-based evaluations.

\section{Methods}

\subsection{Source Data}

This US population-based study used claims data for patients with mCRC that were extracted from two of the Thomson Reuters MarketScan ${ }^{\circledR}$ databases (Commercial Claims and Encounters, and the Medicare Supplemental and Coordination of Benefits [Medicare Supplemental]) for the study period of January 2005 to June 2008. The MarketScan databases include comprehensive, anonymous patient-level, paid and adjudicated medical and pharmacy claims information on 30 million commercially insured patients (both Medicare and supplemental employer-sponsored coverage) spanning 12 national and regional health plans in the USA. The databases capture medical resource use in all settings from physician office visits to hospital stays and outpatient pharmacy claims. The current analysis used only the hospitalisation data contained in the Thomson Reuters MarketScan ${ }^{\circledR}$ databases.

\subsection{Patient Population}

Patients aged $\geq 18$ years were selected on the basis of the International Classification of Disease 9th Revision, Clinical Modification (ICD-9-CM) diagnosis codes for CRC (153.x [excluding 153.5], 154.0, 154.1, 154.8) and distant metastases $(196.0,196.1,196.3,196.5,197 . x$ [excluding 197.5], 198, and 199.0) dated between January 2005 and June 2008. To be eligible for inclusion within the study cohort, the first identified distant metastasis diagnosis had to be no more than 30 days before the first CRC diagnosis. The first distant metastasis diagnosis date was defined as the index date. Patients meeting these eligibility criteria were also required to have shown continuous enrolment for medical and pharmacy benefits for at least 12 months prior to the index date and there was no diagnosis for metastasis during this period. Patients were followed from the index date to death, disenrollment or end of the study period (June, 2008), which- ever occurred first.

\subsection{Outcome Measures}

The main outcome measures investigated in this analysis were length of hospital stay (days) and hospitalisation costs (US\$) per event for AEs associated with the monoclonal antibodies bevacizumab, cetuximab and panitumumab in the treatment of mCRC. AEs for inclusion in the analysis were selected from the "Warnings and precautions" section of the US PI documents [6-8] and the "Special warnings and precautions for use" section of the EMA SmPC [9-11]. Corresponding ICD-9-CM diagnosis codes for each AE were identified based on clinical input from a panel of three oncologists. As there are no designated ICD-9-CM codes for infusion reactions, the signs and symptoms related to infusion reactions were used as a proxy to identify hospital events for infusion reactions [13].

Within the eligible patient cohort, all post-index hospital admission records were examined to identify hospitalisations for selected AEs based on primary discharge diagnoses corresponding to each of the following AEs: arterial thromboembolic event, congestive heart failure (CHF), dermatologic toxicity, fistula, GI perforation, haemorrhage, hypertension, hypomagnesaemia/electrolyte abnormalities, infusion reactions, neutropenia, proteinuria, diffuse parenchymal lung disease/interstitial lung disease (ILD), pulmonary haemorrhage/haemoptysis, reverse posterior leukoencephalopathy syndrome, woundhealing complication and venous thromboembolism.

\subsection{Statistical Analysis}

Average length of stay and reimbursement amount per hospitalisation was calculated from the total number of hospitalisation records for each AE examined in this study. The consumer price index (CPI) was taken into consideration to adjust all cost calculations to 2010 US\$. CPI for 2004 to 2010 ranged from $5.7 \%$ to $9.0 \%$ [14]. Results of the analyses are presented descriptively asmean and median values. To be analytically meaningful, we only studied AEs with data for more than five hospital stays.

\section{Results}

Based on the selection criteria, a total of 12,648 patients with $\mathrm{mCRC}$ met eligibility criteria for inclusion. Patient demographics and clinical characteristics are shown in Table 1. The study population was more or less equally divided by gender ( $54 \%$ men) and was mainly aged 50 years or older $(90.1 \%)$, with a primary diagnosis of colon cancer $(70.1 \%)$ as opposed to rectal cancer. Metastatic deposits were located in the liver in $40.2 \%$ of patients.

Table 2 lists the 12 AEs for which hospital costs per event were examined, namely acute myocardial infarction 
Table 1. Demographic and clinical characteristics of patients with $\operatorname{mCRC}(n=12,648)$.

\begin{tabular}{|c|c|c|}
\hline Variable & $\mathbf{n}$ & Mean or \% \\
\hline Age, mean $\pm \mathrm{SD}$, year & 12,648 & $66.3 \% \pm 13 \%$ \\
\hline Age $<40$ & 240 & $1.9 \%$ \\
\hline $40 \leq$ Age $<50$ & 1012 & $8 \%$ \\
\hline $50 \leq$ Age $<65$ & 4819 & $38.1 \%$ \\
\hline Age $\geq 65$ & 6577 & $52 \%$ \\
\hline Gender: male (vs female) & 6855 & $54.2 \%$ \\
\hline \multicolumn{3}{|l|}{ Geographic region } \\
\hline Northeast & 1189 & $9.4 \%$ \\
\hline North Central & 4364 & $34.5 \%$ \\
\hline South & 4490 & $35.5 \%$ \\
\hline West & 2568 & $20.3 \%$ \\
\hline Unknown & 38 & $0.3 \%$ \\
\hline \multicolumn{3}{|l|}{ Urban vs rural residence } \\
\hline Urban & 10,245 & $81 \%$ \\
\hline Rural & 2353 & $18.6 \%$ \\
\hline Missing & 51 & $0.4 \%$ \\
\hline \multicolumn{3}{|l|}{ Insurance plan type } \\
\hline Comprehensive & 4174 & $33 \%$ \\
\hline HMO & 2125 & $16.8 \%$ \\
\hline PPO & 5059 & $40 \%$ \\
\hline POS-non-capitated & 734 & $5.8 \%$ \\
\hline Other/unknown & 557 & $4.4 \%$ \\
\hline Cancer type: colon (vs rectum) & 8866 & $70.1 \%$ \\
\hline \multicolumn{3}{|l|}{ Location of metastases } \\
\hline Liver & 5084 & $40.2 \%$ \\
\hline Lung & 1796 & $14.2 \%$ \\
\hline Bone & 696 & $5.5 \%$ \\
\hline Brain & 379 & $3 \%$ \\
\hline Others & 4693 & $37.1 \%$ \\
\hline
\end{tabular}

SD: standard deviation; HMO: health maintenance organisations; PPO: preferred provider organisations; POS: point of service plans. 
Table 2. Inpatient hospital length of stay and cost of AEs in patients with mCRC.

\begin{tabular}{|c|c|c|c|c|c|c|c|c|c|}
\hline $\mathbf{A E}$ & & $\mathbf{n}$ & Mean & SD & Median & Min & Max & $\begin{array}{c}\text { Lower } \\
\text { 95\% CI }\end{array}$ & $\begin{array}{c}\text { Upper } \\
\mathbf{9 5 \%} \text { CI }\end{array}$ \\
\hline \multirow{2}{*}{\multicolumn{10}{|c|}{ Acute myocardial infarction }} \\
\hline & & & & & & & & & \\
\hline & Total costs & 89 & 22,395 & 23,380 & 15,223 & 1358 & 128,280 & 17,470 & 27,320 \\
\hline \multirow{2}{*}{\multicolumn{10}{|c|}{ Arterial thromboembolism }} \\
\hline & & & & & & & & & \\
\hline & Total costs & 14 & 40,992 & 50,612 & 18,587 & 2919 & 160,954 & 11,769 & 70,214 \\
\hline \multicolumn{10}{|l|}{ Congestive heart failure } \\
\hline & Total costs & 112 & 12,902 & 18,777 & 8588 & 823 & 116,759 & 9386 & 16,418 \\
\hline \multicolumn{10}{|l|}{ GI perforation } \\
\hline & Total costs & 20 & 66,224 & 80,335 & 34,027 & 2230 & 330,182 & 28,626 & 103,822 \\
\hline \multicolumn{10}{|l|}{ Haemorrhage } \\
\hline & Total costs & 148 & 14,114 & 17,513 & 9433 & 374 & 126,785 & 11,269 & 16,958 \\
\hline \multirow{2}{*}{\multicolumn{10}{|c|}{ Hypertension }} \\
\hline & & & & & & & & & \\
\hline & Total costs & 34 & 14,109 & 23,635 & 6048 & 696 & 128,016 & 5862 & 22,355 \\
\hline \multicolumn{10}{|l|}{ Infusion reactions } \\
\hline & Total costs & 144 & 11,258 & 14,606 & 7559 & 213 & 131,517 & 8852 & 13,664 \\
\hline \multicolumn{10}{|l|}{ Interstitial lung disease } \\
\hline & Total costs & 8 & 26,705 & 27,888 & 19,111 & 8712 & 94,341 & 3390 & 50,020 \\
\hline \multirow{2}{*}{\multicolumn{10}{|c|}{ Stroke }} \\
\hline & & & & & & & & & \\
\hline & Total costs & 134 & 12,586 & 13,847 & 9401 & 1330 & 116,713 & 10,220 & 14,952 \\
\hline \multirow{2}{*}{$\begin{array}{l}\text { Symptoms involving } \\
\text { skin and other } \\
\text { integumentary tissue }\end{array}$} & LOS & 6 & 3.2 & 0.8 & 3.0 & 2 & 4 & 2 & 4 \\
\hline & Total costs & 6 & 6475 & 4014 & 6110 & 1659 & 12,862 & 2263 & 10,687 \\
\hline \multirow{2}{*}{$\begin{array}{l}\text { Venous } \\
\text { thromboembolism }\end{array}$} & LOS & 86 & 5.4 & 6.2 & 4.0 & 1 & 49 & 1 & 14 \\
\hline & Total costs & 86 & 14,332 & 19,555 & 8690 & 1111 & 138,230 & 10,139 & 18,524 \\
\hline \multirow{2}{*}{$\begin{array}{l}\text { Wound healing } \\
\text { complications }\end{array}$} & LOS & 26 & 14.1 & 14.7 & 7.0 & 2 & 48 & 2 & 47 \\
\hline & Total costs & 26 & 36,440 & 41,888 & 21,163 & 503 & 191,300 & 19,521 & 53,359 \\
\hline
\end{tabular}

AE: adverse event; SD: standard deviation; Min: minimum; Max: maximum; CI: confidence interval; LOS: length of stay (days); GI: gastrointestinal. Data are restricted to AEs with data of more than five hospital stays. Adverse events were identified using primary diagnosis code (primary discharge diagnosis; post-index; $n=12,648$ ). Total costs are shown in 2010 US\$. 
(MI), arterial and venous thromboembolism, CHF, GI perforations, haemorrhage, hypertension, infusion reactions, ILD, stroke, symptoms involving skin and other integumentary tissue and wound-healing complications.

As reported in Table 2, the mean and median length of stay associated with the examined AEs ranged from 3.2 to 14.1 days and 2.0 to 11.5 days, respectively. GI perforation incurred the longest median duration of stay (11.5 days) followed by wound-healing complications (7 days), arterial and venous thromboembolism (5.5 and 4 days, respectively) and CHF (4 days). Wound-healing complications and GI perforation had the longest mean length of hospital stay (14.1 days and 13.8 days, respectively).

With respect to the hospital costs of the examined AEs, the CPI-adjusted, mean and median 2010 hospital costs per event ranged from $\$ 6475$ to $\$ 66,224$ and $\$ 6047$ to $\$ 34,027$, respectively (Table 2). GI perforations were associated with the highest costs (mean $\$ 66,224$ and median $\$ 34,027$ ), followed by arterial thromboembolism (\$40,992 and $\$ 18,587)$, wound-healing complications $(\$ 36,440$ and $\$ 21,163)$, ILD (\$26,705 and \$19,111) and acute MI $(\$ 22,395$ and $\$ 15,223)$. By contrast, skin toxicity (mean $\$ 6475$ and median \$6110) and hypertension (mean \$14,108 and median \$6047) were associated with relatively low costs.

\section{Discussion}

The introduction of anti-VEGF and anti-EGFR monoclonal antibodies has broadened the treatment choices for patients with mCRC. Tolerability data from clinical trials of monoclonal antibody treatments for mCRC demonstrate that their respective safety profiles differ from one another.

In this analysis we identified that the costs of selected AEs associated with monoclonal antibody treatments for mCRC depended on the AE in question and that the associated costs varied greatly (from a median of $\$ 6047$ to $\$ 34,027$ and a mean of $\$ 6475$ to $\$ 66,224)$. Across all AEs, GI perforations, arterial thromboembolism, woundhealing complications, ILD and acute MI were among the AEs with the longest lengths of stay and the highest hospitalisation costs. In contrast, skin toxicities and hypertension were associated with the lowest costs. Although some AEs had similar length of stay, they were associated with quite different total costs. For example, both skin and acute MI involved a median of 3 days inpatient stay but incurred median total costs of $\$ 6110$ and $\$ 15,223$, respectively. Length of stay is therefore unlikely to be the only driver of costs, instead variations in treatment intensity and wards where patients were treated are likely to be highly influential factors on treatment costs.

As with all retrospective claims-based analyses, the study findings should be viewed in light of some limitations. It is important to emphasise that the term "AE" has been used throughout this report to describe a complication in patients with mCRC that is consistent with the recognised $\mathrm{AE}$ profile of monoclonal antibody therapies administered to patients with mCRC [6-11]. However, a causal link between any specific $\mathrm{AE}$ and any specific monoclonal antibody therapy was neither investigated nor identified and the frequency of the AEs was not reported by treatments. As this was an economic study, which only evaluated inpatient costs of individual AEs, our aim was simply to estimate the hospitalisation costs of the representative AEs in the mCRC patient population by hospitalisations having the primary discharge diagnosis the same as the AEs, with the assumption that the hospitalisation costs of specific AEs were to be the same regardless of cause.

Additionally, only prevalent AEs were studied here as there were no, or not enough, hospital events identified for some relatively rare AEs. Finally, the difference between mean and median length of stay and hospital costs for some AEs vary substantially, which is generally consistent with the typical distributions of healthcare uses and costs. Both mean and median values were presented to inform appropriate interpretations of the findings.

Our study focused on estimating the hospital cost of monoclonal antibody treatment-related AEs in patients with $\mathrm{mCRC}$ at patient population level not at individual patient (or patient subgroup) level, as the cost of AE information is most likely to be used in economic evaluations at patient population level. The cost of AEs would be more accurate if it were assessed at individual patient (or patient subgroup) level; however, the use of the individual level cost information would be less practical in economic evaluations.

Our study sought only to estimate hospitalisation costs associated with common AEs and thus does not capture the full extent of costs associated with treating these AEs which may also be treated in the outpatient setting. We also made no attempt to measure costs applied to stays in an intensive care unit which could contribute significantly to total costs and also any indirect costs that would further contribute to the total costs incurred by healthcare systems and society. Furthermore, the claims data used in this study represent findings in the US population and may not be wholly transferable as a guide to costs in other countries. Ideally, if the sample size was large enough for each $\mathrm{AE}$, a regression model which controls for covariates would be used to estimate the "expected values" of hospital costs. Due to small sample size, only averages of costs are described. However, standard deviation, minimum and maximum value and $95 \%$ confidence interval have been provided to assess the uncertainty around average estimates. 
This study estimated direct hospitalisation costs per event for prevalent AEs known to be associated with monoclonal antibody treatments for mCRC. The main finding from this study highlights that inpatient costs for those AEs vary substantially. The cost data reported in this study would be of particular value to the economic evaluations of head-to-head comparisons of monoclonal antibody treatments in patients with $\mathrm{mCRC}$.

\section{Authors' Contribution}

AF, ZZ, BB and GGL conceived the study, planned its design and its coordination and drafted the manuscript. $\mathrm{AF}$ and SW organised and built the database and performed the statistical analyses. All authors read and approved the final manuscript.

\section{Acknowledgements}

This study was funded by Amgen Inc. Medical writing assistance was provided by ApotheCom ScopeMedical Ltd, funded by Amgen Inc. All authors had full access to the data and had final responsibility for the decision to submit the manuscript.

\section{REFERENCES}

[1] A. Jemal, R. Siegel, E. Ward, et al., "Cancer statistics, 2009," CA: A Cancer Journal of Clinicians, Vol. 59, No. 4, 2009, pp. 225-249. doi:10.3322/caac.20006

[2] K. R. Yabroff, E. B. Lamont, A. Mariotto, et al., "Cost of Care for Elderly Cancer Patients in the United States," Journal of the National Cancer Institute, Vol. 100, 2008, pp. 630-641. doi:10.1093/jnci/djn103

[3] X. Song, Z. Zhao, B. Barber, et al., "Characterizing medical care by disease phase in metastatic colorectal cancer," Journal of Oncology Practice, Vol. 7, No. 3S, 2011, pp. 25s-30s. doi:10.1200/JOP.2011.000304

[4] J. Ortega, C. E. Vigil and C. Chodkiewicz, "Current Progress in Targeted Therapy for Colorectal Cancer," Cancer Control, Vol. 17, No. 1, 2010, pp. 7-15.

[5] R. M. Goldberg, "Therapy for Metastatic Colorectal Cancer," Oncologist, Vol. 11, No. 9, 2006, pp. 981-987. doi:10.1634/theoncologist.11-9-981

[6] Roche, "Bevacizumab US Prescribing Information," 2010.

[7] Amgen Inc, "Panitumumab US Prescribing Information," 2010.

[8] ImClone Systems Inc. and Bristol-Myers Squibb Company, "Cetuximab US prescribing information," 2010.

[9] Roche, "Bevacizumab Summary of Product Characteristics," 2010.

[10] Amgen Inc, "Panitumumab Summary of Product Characteristics," 2010.

[11] ImClone Systems Inc. and Bristol-Myers Squibb Company, "Cetuximab Summary of Product Characteristics," 2010.

[12] G. Dranitsaris, J. Maroun and A. Shah, "Estimating the Cost of Illness in Colorectal Cancer Patients Who Were Hospitalized for Severe Chemotherapy-Induced Diarrhea," Canadian Journal of Gastroenterology, Vol. 19, No. 2, 2005, pp. 83-87.

[13] K. A. Foley, P. F. Wang, B. L. Barber, et al., "Clinical and Economic Impact of Infusion Reactions in Patients with Colorectal Cancer Treated with Cetuximab," Annals of Oncology, Vol. 21, No. 7, 2010, pp. 1455-1461. doi:10.1093/annonc/mdp535

[14] "US Bureau of Labor Statistics," 2010 http://www.bls.gov/cpi/ 